\title{
Análise de Históricos de Contextos para Classificação do Estresse Mental em Situações Reais por meio da Variabilidade da Frequência Cardíaca
}

\author{
Rodrigo Simon Bavaresco, Jorge Luis Victória Barbosa \\ Universidade do Vale do Rio dos Sinos (UNISINOS) \\ Av. Unisinos, 950 - Cristo Rei, São Leopoldo - RS, 93022-000 \\ rsbavaresco@gmail.com, jbarbosa@unisinos.br
}

\begin{abstract}
Mental stress is a major cause of physical and emotional health problems in the world. In this way, it becomes strategic to measure daily stress throught physiology, using these measures in applications that aim individuals' well-being. However, daily motion influences physiological data collection, producing noise. This work collects and analyzes the contexts histories of 5 individuals with the purpose of classifying mental stress through heart rate variability. As a scientific contribution, the method identifies and removes noise, as well as shows a relation of the stress levels and the location in which they were measured. The best performance, among three machine learning algorithms was obtained from Support Vector Machine classifier, resulting in $82 \%$ of accuracy.
\end{abstract}

Resumo. O estresse mental é um dos principais causadores de problemas na saúde física e emocional. Desta forma, torna-se estratégico mensurar o estado de estresse diário por meio fisiológico a fim de utilizá-lo em aplicações que visem o bem-estar do indivíduo. Entretanto, as movimentações diárias influenciam na coleta de dados fisiológicos, causando ruído. Este trabalho coleta e analisa os históricos de contextos de 5 indivíduos com o intuito da classificação do estresse mental por meio da variabilidade da frequência cardíaca. Como contribuição científica, o método visa identificar e remover ruído bem como mostrar uma relação entre os níveis de estresse e a localização em que foram mensurados. A melhor performance obtida, dentre três algoritmos de aprendizado de máquina utilizados foi do classificador Support Vector Machine, resultando em $82 \%$ de acurácia.

\section{Introdução}

O estresse mental é um dos principais causadores de problemas na saúde física e emocional no mundo, sendo considerado como "Epidemia do Século 21" pelo World Health Organization [Fink 2017]. De forma crônica, o estresse mental está relacionado diretamente com o desenvolvimento de desordens mentais, tais como ansiedade e depressão. Além disso, ele está associado a doenças cardiovasculares e autoimunes, diabetes e infecções respiratórias [Cohen et al. 2012, Pereira et al. 2017]. Portanto, torna-se estratégico levar em consideração o estado de estresse diário no desenvolvimento de aplicações que visem o bem-estar do indivíduo. 
O Sistema Nervoso Autônomo (SNA), subdividido em Sistema Nervoso Simpático (SNS) e Sistema Nervoso Parassimpático (SNP), é responsável por coordenar a atividade cardiovascular, digestiva e respiratória. Além disso, o SNA controla respostas involuntárias à estímulos externos, tais como respostas do corpo humano em situações ameaçadoras, conhecido como o fenômeno fight-or-flight [Feher 2012]. Desta forma, excitações como o estresse mental influenciam o nível da atividade autonômica causando uma variedade de respostas fisiológicas, como o aumento ou a redução da Frequência Cardíaca (FC) e da Variabilidade da Frequência Cardíaca (VFC) [Quintana et al. 2016]. Conceitua-se a VFC como a variação ao longo do tempo entre batimentos cardíacos consecutivos. Ela é obtida de forma não invasiva por meio da leitura dos sinais de um eletrocardiograma (ECG), com duração de minutos ou horas. No ECG, a diferença entre duas ondas R é calculada, denominando-se esta diferença de intervalos RR [Peltola 2012].

Nas últimas décadas, o desenvolvimento tecnológico relacionado a dispositivos de hardware e software sofreu tanta expansão que dispositivos móveis - smartphones, tablets e wearables - são utilizados de forma contínua [Barbosa 2015]. Em consequência disso, o smartphone está grande parte do tempo junto ao indivíduo e compreende uma plataforma técnica poderosa, ou seja, apresenta extenso poder computacional, extensa capacidade de comunicação e diversos sensores incorporados [Gravenhorst et al. 2015]. Além disso, quando utilizado juntamente com wearables, torna-se efetivo na manipulação de dados em tempo real sobre o comportamento e fisiologia dos indivíduos.

Neste cenário, avaliações pessoais provenientes dos indivíduos podem ser necessárias, principalmente em relação ao seu estado mental percebido. Desta forma, diversos métodos tem sido explorados, tais como questionários e auto-relatos retrospectivos. Entretanto, as aplicações devem-se ater a fornecer auto-relatos disponíveis no momento em que um evento de interesse ocorrer. Assim, o método Ecological Momentary Assessment (EMA) visa minimizar o viés de memória e maximizar a validade ecológica, permitindo o estudo do comportamento em contextos no decorrer das situações [Shiffman et al. 2008].

A computação sensível ao contexto provém uma forma de organização destes contextos, tanto para coleta, quando para armazenamento e processamento. Ela fornece uma fonte adicional e mais completa de informação, tornando as aplicações cientes das interações entre indivíduos e seu ambiente [da Rosa et al. 2016]. Caracteriza-se o contexto como qualquer informação que possa ser usada para descrever as circunstâncias relativas a uma entidade, tais como pessoas, locais ou objetos [Barbosa 2015].

Este trabalho apresenta a coleta de dados sensíveis ao contexto diante das atividades diárias de indivíduos com o intuito da classificação do estresse mental. As atividades diárias proporcionam a coleta de dados frente a situações reais, o que difere de estudos conduzidos por estressores específicos, normalmente executados em laboratório [Kusserow et al. 2013]. Entretanto, devido à estas situações a presença de artefatos que causam ruído nos dados fisiológicos é constante [Peltola 2012, Kusserow et al. 2013]. Desta forma, como contribuição científica o método apresentado usa dados sensíveis ao contexto a fim de: (i) identificar e remover ruído, tais como a movimentação e as atividades físicas; (ii) mostrar uma relação dos níveis de estresse e a localização em que foram mensurados. 
Este artigo está organizado em cinco seções. Na seção 2 apresentam-se os trabalhos relacionados e na seção 3 descrevem-se os materiais e métodos utilizados nesta pesquisa. A seção 4 fornece os resultados encontrados. Por fim, a seção 5 apresenta as considerações finais.

\section{Trabalhos Relacionados}

Nesta seção apresentam-se os trabalhos relacionados com esta pesquisa. Para busca dos trabalhos, considerou-se como principal referência um estudo sistemático previamente realizado pelo autor. Este estudo mapeou o estado da arte da computação ubíqua aplicada à fisiologia, enfatizando à saúde mental. Sob os resultados, aplicaram-se critérios de inclusão tais como o uso de contexto em situações reais e a classificação de estresse. Os trabalhos resultantes serão apresentados e comparados a seguir.

Tabela 1. Comparativo entre os trabalhos relacionados

\begin{tabular}{ccccc}
\hline & $\begin{array}{c}\text { Medidas } \\
\text { Fisiológicas }\end{array}$ & Elementos do Contexto & $\begin{array}{c}\text { Remoção de } \\
\text { Ruído }\end{array}$ & $\begin{array}{c}\text { Estresse x } \\
\text { Localização }\end{array}$ \\
\hline [Peternel et al. 2012] & EDA & $\begin{array}{c}\text { Ligação, Localização, } \\
\text { Atividade, Áudio, PC } \\
\text { Hora, Dia, Localização, } \\
\text { Clima, Atividade }\end{array}$ & $\begin{array}{c}\text { Atividade } \\
\text { Física }\end{array}$ & $\begin{array}{c}\text { Casa e } \\
\text { Trabalho } \\
\text { Casa e }\end{array}$ \\
[Alharthi et al. 2017] & VFC & Não & Trabalho \\
[Gjoreski et al. 2017] & BVP, EDA, & Hora, Dia, Atividade & Física & Não \\
[Mishra et al. 2018] & VFC & Hora, Dia da semana e & Fisiológico & Não \\
\hline
\end{tabular}

Segundo Peternel et al. 2012, os autores propuseram o primeiro trabalho que reconhece estresse utilizando contexto, com uso da EDA (Electrodermal Activity) apenas nos aspectos de validação. As relações com estresse foram feitas envolvendo localizações específicas, isto é, somente na casa ou no trabalho. Mishra et al. 2018 utilizaram dispositivo de pulso para investigar o uso do contexto, removendo ruído baseado nos limites da fisiologia humana. Embora tenham relacionado o contexto com a atividade do indivíduo, não consideraram a localização.

Alharthi et al. 2017, propuseram um modelo com uso de sensor cardíaco e smartphone. Entretanto, a atividade não foi reconhecida por meio dos sensores dos dispositivos, mas com questionários. Gjoreski et al. 2017 coletaram diversos dados fiosológicos, tais como EDA, BVP (Blood Volume Pulse), HR (Heart Rate), IBI (Inter-beatinterval) e ST (Skin Temperature). Os autores removeram ruído por meio de uma abordagem com aprendizado de máquina. Entretanto, não utilizaram sensores de localização, nem relações envolvendo as mesmas.

A fim de comparar os trabalhos relacionados, definiram-se 4 critérios como podese observar na Tabela 1. Os critérios de comparação são: (i) medidas fisiológicas, que ilustra as medidas usadas para avaliar o SNA; (ii) elementos do contexto, que apresenta quais os dados foram extraídos dos dispositivos, caracterizando as interações com o ambiente; (iii) remoção de ruído, necessário para diferenciar alterações fisiológicas provenientes das atividades físicas daquelas provenientes das psicológicas [Kusserow et al. 2013]; (iv) relação entre estresse e localização, que fornece um método de retirar informação contextual significativa em relação ao ambiente. Desta forma, salienta-se que os trabalhos apresentados não combinam efetivamente os dois últimos critérios, com exceção do 
trabalho de Peternel et al. 2012. Entretanto, os autores não consideraram a flexibilidade de localização proveniente do cotidiano para a relação, possuindo os dados direcionados apenas para a Casa e o Trabalho do indivíduo.

\section{Materiais e Métodos}

Nesta seção apresentam-se os aspectos relacionados com a metodologia de pesquisa e avaliação utilizada por este trabalho.

\subsection{Descrição da Aplicação}

Uma aplicação para smartphone foi desenvolvida para ser utilizada juntamente com um sensor de frequência cardíaca durante as atividades diárias dos indivíduos. No desenvolvimento, implementou-se a coleta contínua de diversos dados que compreendem o contexto. Cada dado possui uma finalidade distinta, que combinados fornecem informações relevantes para a classificação de estresse. Os contextos coletados foram enviados para um servidor na nuvem e armazenados em um banco de dados, formando séries temporais de contextos. A sequência de séries temporais observadas levam o nome de históricos de contextos [da Rosa et al. 2016].

Constituem o contexto do indivíduo seis entidades principais: Identidade, que representa o indivíduo de forma única; Fisiologia, que representa as leituras dos batimentos cardíacos; Atividade, que apresenta o tipo de movimentação do indivíduo naquele contexto, tais como imóvel, caminhando, correndo, no veículo e de bicicleta; Localização, que fornece dados brutos para a computação da atividade, bem como para a relação com níveis de estresse; DataHora, que identifica o decorrer do tempo; e por fim a entidade Avaliação, que é o estado de estresse percebido pelo indivíduo em determinados contextos. Além disso, a entidade Classificação também faz parte do contexto. Ela é proveniente da computação da VFC e da classificação do estresse mental.

As operações básicas que permitem uma usabilidade adequada da aplicação foram implementadas, tais como uma tela de login e uma tela de pareamento do wearable. Além disso, uma tela para a avaliação EMA foi implementada. Com o intuito de identificar a atividade do indivíduo em cada contexto, de acordo com a entidade Atividade, implementou-se o consumo do serviço Google Location Services ${ }^{1}$. Este, por sua vez, utiliza os recursos do smartphone tais como GPS, acelerômetro e giroscópio para inferir a atividade do usuário. Para a leitura da frequência cardíaca e dos intervalos RR, codificouse um serviço específico para as conexões Bluetooth Low Energy do wearable. Uma vez que a coleta dos dados é realizada de forma contínua, os contextos foram armazenados na memória do smartphone por 5 minutos e enviados em lote para o servidor nesse intervalo. No servidor, implementaram-se módulos a fim de fornecer serviços HTTP, autenticar cada requisição, gerir e persistir os dados de contexto, mantendo o histórico do indivíduo.

\subsection{Coleta dos Dados}

No experimento realizado, a aplicação foi utilizada por 5 voluntários durante 8 dias cada, totalizando 40 dias de uso total. Cada voluntário fez uso com seu próprio smartphone durante suas atividades cotidianas, variando de 4 até 8 horas diárias. Os voluntários são todos homens adultos, saudáveis, não fumantes, com idade média $28 \pm 5$. Eles foram

\footnotetext{
${ }^{1}$ https://developer.android.com/training/location/
} 
solicitados a não ingerir bebidas alcoólicas ou tomar medicamentos caso fossem usar a aplicação, visto que estes fatores influenciam a VFC [Quintana et al. 2016]. O wearable com sensor de frequência cardíaca utilizado foi o mesmo para todos os voluntários, denominado Polar H7. Ele forneceu a frequência cardíaca e os intervalos RR, sendo considerado aceitável sua utilização quando comparado à coleta de dados cardíacos do ECG.

Inicialmente, cada voluntário cadastrou-se na aplicação e realizou o pareamento do wearable. Na sequência, foi explicada a necessidade dos indivíduos realizarem avaliações periódicas de estresse ou quando se sentissem estressados, por meio do EMA. Além disso, os voluntários tornaram-se cientes de que a aplicação os notificaria a cada 2 horas de uso, a fim de realizar a avaliação. Entretanto, a aplicação não considerou a avaliação obrigatória. Por fim, os voluntários puderam seguir normalmente suas rotinas.

\subsection{Rotulagem}

As avaliações do estado de estresse realizadas pelos voluntários por meio do EMA foram utilizadas para rotular as séries temporais de acordo com o estresse percebido. Em cada avaliação, o voluntário pode selecionar um número numa escala de zero (0) até quatro (4), onde o primeiro valor significa "não estressado" e o último "muito estressado".

Devido a falta de uma definição concreta da duração do estresse nas atividades diárias [Kusserow et al. 2013], considerou-se uma janela de 10 minutos antes e depois de cada avaliação, aproximando-se do método proposto em [Gjoreski et al. 2017]. Desta forma, as séries temporais presentes neste intervalo foram rotuladas com o estado "não estressado" caso a avaliação recebida seja do nível zero (0) ou um (1), sendo que os demais níveis foram considerados como o estado "estressado".

\subsection{Segmentação e Pré-processamento}

A segmentação das séries temporais foi realizada considerando a norma de tempo curto (short-term norm) com 5 minutos de duração [Quintana et al. 2016]. Para a segmentação, todos os contextos coletados seguiram uma sequência válida no tempo que respeite a fisiologia humana. Ou seja, foram descartadas séries temporais que apresentaram diferença maior do que 2 segundos entre dois contextos consecutivos.

O pré-processamento das séries temporais foi subdividido em duas etapas. A primeira etapa visou remover ruído causado pela presença de movimentação e atividades físicas. Desta forma, a partir da atividade do indivíduo em cada contexto, a lógica aplicada na Equação 1 determinou se a série temporal torna-se válida para classificação do estresse.

$$
\sum_{i=1}^{n}\left(\alpha_{i}+\beta_{i}\right) \geq n \cdot 0.9 \wedge C_{\alpha, \beta} \geq 60
$$

onde,

$n \quad=$ tamanho da amostra da série temporal, short-term norm

$\alpha=$ contextos com a atividade No Veiculo, $\alpha \in \mathbb{N}^{[0,1]}$

$\beta=$ contextos coma a atividade Imóvel, $\beta \in \mathbb{N}^{[0,1]}$

$C_{\alpha, \beta}=$ grau de confiança da atividade, $C \in \mathbb{N}^{[0,100]}$ 
A Equação 1 mostra que uma série temporal válida deve conter ao menos $90 \%$ de seus contextos com uma combinação das atividades Imóvel ou No Veículo, sendo que o grau de confiança de cada atividade deve ser maior ou igual a 60. Além disso, a série temporal torna-se inválida e é descartada caso apresente mais de $10 \%$ de contextos com valores inválidos na atividade ou na fisiologia, como por exemplo, valores zerados do sensor cardíaco ou atividade desconhecidas.

A segunda etapa visou eliminar artefatos fisiológicos, tal como batidas ectópica ou ainda, dados caracterizados pelo sensor mal conectado. Portanto, as medidas da VFC menores do que 0,3 segundos ou maiores do que 2 segundos foram removidas. Adicionalmente, os valores dos intervalos da VFC que diferiram mais do que 25\% dos 50 intervalos anteriores ou sucessivos foram interpolados utilizando interpolação de spline cúbica. A estratégia de interpolação visa não excluir os intervalos, possibilitando manter o tamanho próximo da série temporal original.

\subsection{Extração e Seleção de Características}

As características foram extraídas a partir dos intervalos RR de cada série temporal préprocessada, conforme a Tabela 2. Elas foram computadas no domínio de tempo e no domínio de frequência. As características no domínio de tempo foram obtidas por meio de cálculos estatísticos. As características do domínio de frequência foram obtidas através da computação da Transformada Rápida de Fourier das séries dos intervalos RR, uniformemente reamostrados a $4 \mathrm{~Hz}$ utilizando interpolação de spline cúbica.

Tabela 2. Características no domínio de tempo e frequência

\begin{tabular}{lcc}
\hline Característica & Unid. & Fórmula \\
\hline Domínio de Tempo & & \\
\hline Average of RR interval (AVRR) & $m s$ & $\overline{R R}=\frac{1}{N} \sum_{j=1}^{N} R R_{j}$ \\
Standard Deviation of RR intervals (SDRR) & $m s$ & $\sqrt{\frac{1}{N-1} \sum_{j=1}^{N}\left(R R_{j}-\overline{R R}\right)^{2}}$ \\
Standard Deviation of the Average RR intervals (SDARR) & $m s$ & $\sqrt{\frac{1}{N-1} \sum_{j=1}^{N}\left(R R_{1_{j}}-\overline{R R_{1}}\right)^{2}}$ \\
Root Mean Square of RR interval Diff. (RMSSD) & $m s$ & $\sqrt{\frac{1}{N-1} \sum_{j=1}^{N}\left(R R_{j+1}-R R_{j}\right)^{2}}$ \\
\% of RR intervals that differ by more than 50 $\mathbf{~ m s}$ (pRR5) & $\%$ & $\frac{N N 50}{N-1} \times 100 \%$ \\
\hline Domínio de Frequência & & \\
\hline Low Frequency $(\boldsymbol{L F})$ & $m s^{2}$ & Potência espectral entre 0,04 e $0,15 \mathrm{~Hz}$ \\
High Frequency $(\mathbf{H F})$ & $m s^{2}$ & Potência espectral entre 0,15 e $0,4 \mathrm{~Hz}$ \\
Ratio LF/HF & $m s^{2}$ & Razão entre LF e HF \\
\hline
\end{tabular}

A seleção visou identificar as características mais relevantes para treinamento dos modelos de aprendizado de máquina. Para tal, utilizou-se um algoritmo projetado em torno (wrapper) do algoritmo de classificação Random Forest [Kursa, M B. and Rudnicki, W. R. 2010]. O algoritmo utilizado remove iterativamente as características menos relevantes, que são provadas por um teste estatístico. Conforme a Tabela 2, oito características foram computadas inicialmente. Dentre elas, a característica Standard Deviation of the Average RR intervals (SDARR) foi dada como irrelevante pelo algoritmo. Consequentemente, ela foi removida das etapas de análise, treino e teste.

\subsection{Análise Estatística e Classificação}

A análise estatística visou verificar se existe diferença significativa entre os estados de estresse rotulados como "não estressado" e "estressado" a partir das características ex- 
traídas, nomeadas de variáveis. Para esta análise, foram usados os dados dos 5 voluntários agrupados e somente as séries temporais válidas. Inicialmente, buscou-se identificar se a relação dos dados apresenta distribuição normal, aplicando o teste Shapiro-Wilk. Caso positivo, executa-se o Teste t de Student, e caso contrário, executa-se o teste Wilcoxon Signed Rank. A significância estatística levada em consideração foi $\alpha=0,05$.

$\mathrm{Na}$ classificação do estresse, três algoritmos de aprendizado de máquina supervisionado foram treinados e testados: Naive Bayes (NB), K-Nearest Neighbors (KNN) e Support Vector Machines (SVM). A mesma metodologia de treino e teste foi utilizada para cada algoritmo. Primeiro, os dados totais foram subdivididos em $75 \%$ para a amostra de treino de $25 \%$ para a de teste. No experimento de treino, uma busca em grade com validação cruzada 10 -fold foi realizada permitindo melhor ajustar cada modelo de classificação. No experimento de teste, os três modelos foram avaliados pela performance de classificação sob o conjunto de dados desconhecido.

Para avaliar cada classificador, calcularam-se métricas estatísticas: Sensitividade ou Recall, que se refere a identificar corretamente indivíduos sob estresse; Especificidade, que se refere a identificar corretamente os indivíduos que não estão sob estresse; Precisão, que é uma medida que nos diz qual a proporção de indivíduos que foram identificados como estressados e na verdade, estavam estressados e Acurácia, que é uma medida de previsões corretas de indivíduos estressados e não estressados. Por fim, também é apresentado o F1-Score, que é a média ponderada da Precisão e do Recall.

\section{Resultados}

A partir da execução dos métodos descritos na seção anterior, foram extraídas um total de 304 séries temporais válidas de 5 minutos cada. O rótulo do estado "estressado" é apresentado em $40 \%$ das séries e o restante apresenta o rótulo "não estressado". A seguir, mostram-se os resultados obtidos por meio dos métodos de análise estatística e classificação, além da relação do estresse com a localização.

\subsection{Variabilidade da Frequência Cardíaca}

Para esta análise, tornou-se necessário subdividir os dados totais em duas amostras de mesmo tamanho. Desta forma, os primeiros $80 \%$ das séries válidas foram utilizados, que compreendem $40 \%$ de séries rotuladas com o estado "não estressado" e $40 \%$ como "estressado". O teste Shapiro-Wilk possibilitou identificar que a distribuição dos dados para todas as variáveis não segue uma distribuição normal $(p<0.02)$. Desta forma, aplicou-se o teste Wilcoxon Signed Rank sob amostras dependentes.

A Tabela 3 apresenta os resultados estatísticos do experimento nas métricas Mediana e Amplitude Interquartil (AI) de cada variável, de acordo com o rótulo dos dados realizados pelos voluntários, onde AI é a diferença entre o quartil superior e o inferior. Além disso, o resultado do $p$-valor é apresentado para cada variável. A hipótese nula criada verifica se as duas amostras não possuem diferença estatística, ou seja, se são idênticas. Portanto, a hipótese nula foi rejeitada para todas as variáveis, de acordo com o $p$-valor e a significância de $\alpha=0,05$. Consequentemente, identifica-se que as variáveis apresentam diferença estatística. Entretanto, a menor diferença significativa foi encontrada na razão LF/HF. 
Adicionalmente, os valores dos estados "não estressado" e "estressado" ilustram que todas as variáveis possuem valores numéricos inferiores no estado de estresse, com exceção da razão LF/HF. A razão LF/HF representa o balanço simpato-vagal, ou seja, uma medida da atividade do sistema nervoso simpático [Quintana et al. 2016]. Embora ela não apresente diferença significativa, o maior valor em estresse indica uma maior atividade simpática nestas situações. Desta forma, estes resultados se assemelham aos encontrados na literatura [Pereira et al. 2017].

Tabela 3. Resultados do teste Wilcoxon Signed Rank para cada variável

\begin{tabular}{lccc}
\hline \multirow{2}{*}{ Variável } & \multicolumn{2}{c}{ Mediana (AI) } & p-valor \\
\cline { 2 - 3 } & Não Estressado & Estressado & \\
\hline $\operatorname{AVRR}(m s)$ & $829,6(96,7)$ & $691,6(144,8)$ & $p<0,0001$ \\
$\operatorname{SDRR}(m s)$ & $68,5(26,5)$ & $46,5(26,2)$ & $p<0,0001$ \\
$R M S S D(m s)$ & $47,43(22,2)$ & $27(19,1)$ & $p<0,0001$ \\
$p R R 50(\%)$ & $26,0(21,7)$ & $6,3(9)$ & $p<0,0001$ \\
$H F\left(m s^{2}\right)$ & $117,8(105,8)$ & $37,8(43,8)$ & $p<0,0001$ \\
$L F\left(m s^{2}\right)$ & $242,1(198,8)$ & $92,5(98,9)$ & $p<0,0001$ \\
$L F / H F\left(m s^{2}\right)$ & $2,1(1,3)$ & $2,4(1,8)$ & $p=0,0106$ \\
\hline
\end{tabular}

\subsection{Performance de Classificação}

As séries temporais válidas foram subdivididas em amostra de treino e de teste, onde cada algoritmo de classificação foi utilizado para o experimento de treino e posteriormente para o de teste. No experimento de teste, $60 \%$ da amostra compreendeu o estado "não estressado" e $40 \%$ o estado "estressado". Como resultado do experimento, uma matriz de confusão foi gerada para cada classificador. Analisando cada matriz, as métricas para avaliar a performance de cada classificador puderam ser calculadas.

A Tabela 4 apresenta os resultados das métricas de cada classificador. Estes resultados mostram que, embora o SVM possua precisão e especificidade maiores que $90 \%$, buscando um balanço entre precisão e sensitividade, ilustrado pelo F1-Score, tanto o KNN quanto o SVM apresentam o resultado de $75 \%$. Desta forma, a segunda métrica considerada relevante para análise torna-se a acurácia, que é a razão de previsões corretas para o total de previsões feitas. Neste caso, o SVM possui o maior valor. Portanto, a melhor performance de classificação foi obtida com o algoritmo SVM.

Tabela 4. Resultados estatísticos da performance de classificação

\begin{tabular}{lrrrrr}
\hline Classificador & Sens. & Espec. & Prec. & Acur. & F1 \\
\hline Naive Bayes & 0,77 & 0,62 & 0,58 & 0,68 & 0,66 \\
K-Nearest Neighbors & 0,74 & 0,84 & 0,76 & 0,80 & 0,75 \\
Support Vector Machine & 0,64 & 0,95 & 0,90 & 0,82 & 0,75 \\
\hline
\end{tabular}

\subsection{Estresse e Localização}

Além de possibilitar a remoção de ruído, os dados sensíveis ao contexto coletados tornaram possível relacionar o estado de estresse computado com a localização em que foram 
mensurados. Desta forma, a aplicação não restringe-se a lugares específicos, tais como a casa ou trabalho, e torna-se genérica para qualquer localização frequentada.

A Figura 1 apresenta a relação entre 6 localizações frequentadas pelo voluntário que mais gerou dados durante o uso da aplicação. Mostram-se os minutos de permanência em cada localização diante apenas das séries temporais válidas, sendo evidenciado o percentual de tempo em que o indivíduo apresentou estresse.

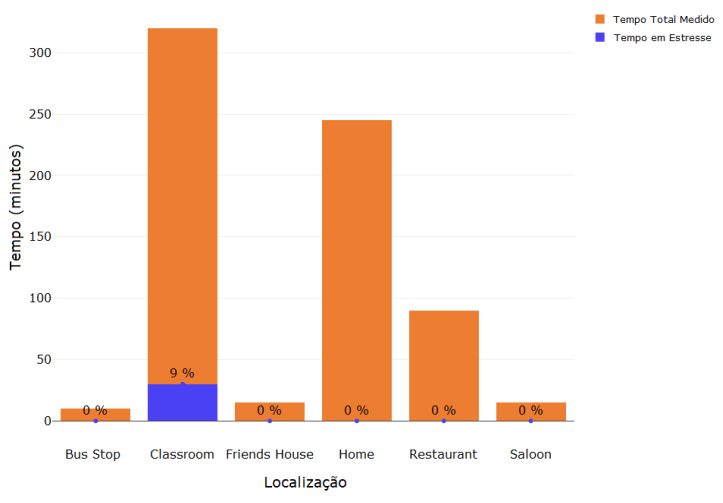

Figura 1. Relação entre o estresse e a localização obtida

\section{Considerações Finais}

Este trabalho apresentou a coleta de dados sensíveis ao contexto com o intuito da classificação do estresse mental. Estes dados possibilitaram identificar e remover ruído da frequência cardíaca, tais como a movimentação e as atividades físicas, bem como mostrar uma relação dos níveis de estresse e a localização em que foram mensurados.

Além disso, a coleta de dados durante o cotidiano possibilitou analisar estatisticamente estados de "estresse" com o intuito de verificar diferenças significativa nas características extraídas da VFC. Por fim, foram treinados três algoritmos de aprendizado de máquina a fim de classificar o estado de estresse mental. O algoritmo com melhor performance nos testes efetuados foi o SVM, que apresentou acurácia de $82 \%$ e F1-Score de $75 \%$. Entretanto, algumas limitações são observadas, tais como o baixo número de indivíduos para a coleta de dados bem como a presença somente de indivíduos do sexo masculino.

Como trabalhos futuros, identifica-se a possibilidade de incorporar os métodos apresentados em aplicações que visem o bem-estar dos indivíduos. Assim, podem ser exploradas técnicas de intervenções a medida que identificam-se momentos de estresse no cotidiano, visando reduzir os efeitos negativos do estresse mental.

\section{Agradecimentos}

Os autores agradecem à FAPERGS, ao CNPq, à Coordenação de Aperfeiçoamento de Pessoal de Nível Superior (CAPES) - Código de Financiamento 001, e à Universidade do Vale do Rio dos Sinos (Unisinos) pelo apoio ao desenvolvimento desse trabalho. 


\section{Referências}

Alharthi, R., Alharthi, R., Guthier, B., and El Saddik, A. (2017). CASP: context-aware stress prediction system. Multimedia Tools and Applications, pages 1-21.

Barbosa, J. L. V. (2015). Ubiquitous computing: Applications and research opportunities. In 2015 IEEE International Conference on Computational Intelligence and Computing Research (ICCIC), pages 1-8. IEEE.

Cohen, S., Janicki-Deverts, D., Doyle, W. J., Miller, G. E., Frank, E., Rabin, B. S., and Turner, R. B. (2012). Chronic stress, glucocorticoid receptor resistance, inflammation, and disease risk. Proceedings of the National Academy of Sciences, 109(16):59955999.

da Rosa, J. H., Barbosa, J. L., and Ribeiro, G. D. (2016). ORACON: An adaptive model for context prediction. Expert Systems with Applications, 45:56-70.

Feher, J. (2012). Autonomic Nervous System. In Quantitative Human Physiology, pages 403-416. Elsevier.

Fink, G. (2017). Stress: Concepts, Definition and History. Reference Module in Neuroscience and Biobehavioral Psychology, (January):0-9.

Gjoreski, M., Luštrek, M., Gams, M., and Gjoreski, H. (2017). Monitoring stress with a wrist device using context. Journal of Biomedical Informatics, 73:159-170.

Gravenhorst, F., Muaremi, A., Bardram, J., Grünerbl, A., Mayora, O., Wurzer, G., Frost, M., Osmani, V., Arnrich, B., Lukowicz, P., and Tröster, G. (2015). Mobile phones as medical devices in mental disorder treatment: an overview. Personal and Ubiquitous Computing, 19(2):335-353.

Kursa, M B. and Rudnicki, W. R. (2010). Feature Selection with the Boruta Package. Journal of Statistical Software, 36(11):1-13.

Kusserow, M., Amft, O., and Tröster, G. (2013). Monitoring stress arousal in the wild. IEEE Pervasive Computing, 12(2):28-37.

Mishra, V., Hao, T., Sun, S., Walter, K. N., Ball, M. J., Chen, C.-H., and Zhu, X. (2018). Investigating the Role of Context in Perceived Stress Detection in the Wild. In UbiComp '18, pages 1708-1716, New York, New York, USA. ACM Press.

Peltola, M. (2012). Role of editing of r-r intervals in the analysis of heart rate variability. Frontiers in Physiology, 3:148.

Pereira, T., Almeida, P. R., Cunha, J. P., and Aguiar, A. (2017). Heart rate variability metrics for fine-grained stress level assessment. Computer Methods and Programs in Biomedicine, 148:71-80.

Peternel, K., Poga, Tavčar, R., and Kos, A. (2012). A presence-based context-aware chronic stress recognition system. Sensors (Switzerland), 12(11):15888-15906.

Quintana, D. S., Alvares, G. A., and Heathers, J. A. J. (2016). Guidelines for reporting articles on psychiatry and heart rate variability (graph): recommendations to advance research communication. Translational Psychiatry, 6(5):e803-e803.

Shiffman, S., Stone, A. A., and Hufford, M. R. (2008). Ecological Momentary Assessment. Annual Review of Clinical Psychology, 4(1):1-32. 